\title{
La Fable des abeilles de Bernard Mandeville
}

L'exploitation de son prochain comme fondement de la civilisation

Hervé Mauroy

\section{CpenEdition}

\section{Journals}

Édition électronique

URL : http://journals.openedition.org/ress/843

DOI : $10.4000 /$ ress.843

ISSN : 1663-4446

Éditeur

Librairie Droz

\section{Édition imprimée}

Date de publication : 15 octobre 2011

Pagination : 83-110

ISBN : 978-2-600-01551-6

ISSN : 0048-8046

\section{Référence électronique}

Hervé Mauroy, "La Fable des abeilles de Bernard Mandeville », Revue européenne des sciences sociales [En ligne], 49-1 | 2011, mis en ligne le 01 janvier 2015, consulté le 10 décembre 2020. URL : http:// journals.openedition.org/ress/843; DOI : https://doi.org/10.4000/ress.843 


\title{
LA FABLE DES ABEILLES \\ DE BERNARD MANDEVILLE \\ L'EXPLOITATION DE SON PROCHAIN \\ COMME FONDEMENT DE LA CIVILISATION
}

\author{
HERVÉ MAUROY \\ Université de Valenciennes \\ herve.mauroy@univ-valenciennes.fr
}

\begin{abstract}
Résumé. Dans les textes rassemblés dans la Fable des abeilles, Bernard Mandeville a proposé une théorie de l'évolution culturelle en s'inspirant des selfish systems of morals du XVII siècle (La Rochefoucauld, Esprit, Nicole, Bayle...). Il y explique que les gens poussés par les « plus méchants » ont mis sur pied une superstructure morale et juridique qui, en les poussant à collaborer entre eux, a créé et fait progresser la civilisation. Cet article vise d'abord à explorer spécifiquement le rôle joué par les «pires des hommes ». Mandeville a proposé pour ce faire une ingénieuse argumentation théorique à partir de l'idée selon laquelle la plupart des mandatés et mandataires ne cessent pas de chercher à se berner les uns les autres du fait de l'existence entre eux d'asymétries informationnelles.
\end{abstract}

Mots-clés: Mandeville; ordre spontané; économie des conventions; systèmes égoïstes de la morale; théorie des jeux

\begin{abstract}
In the texts which compose the Fable of the Bees, Bernard Mandeville sets forth a theory of cultural evolution drawing his inspiration from the selfish systems of morals of the 17 th century (La Rochefoucauld, Esprit, Nicole, Bayle...). He holds that the people pushed by « the very worst » among themselves managed to create and develop their civilisation by setting up a moral and legal superstructure prompting them to « collaborate » with one another. The aim of this article is to study specifically the role performed by those referred to as the very worst among men. In order to do so, Mandeville comes up with an ingenious theoretical argumentation starting from the assumption that most of the agents and principals keep trying to double-cross one another because of the existence of informational asymmetries between them.
\end{abstract}

Keywords: Mandeville; spontaneous order; economics of convention; selfish systems of morals; game theory 
It is to no purpose that the proud and unfeeling landlord views his extensive fields, and without a thought for the wants of his brethren, in imagination consumes himself the whole harvest that grows upon them [...] They are led by an invisible hand. (Adam Smith, The theory of the moral sentiments.)

Il fallut, pour son avantage, se montrer autre que ce qu'on était en effet. Être et paraître devinrent deux choses tout-à-fait différentes; et de cette distinction sortirent le faste imposant, la ruse trompeuse. (Jean-Jacques Rousseau, Discours sur l'origine et les fondements de l'inégalité parmi les hommes.)

Bernard Mandeville (I670-I733), «analyste» des motivations intérieures des actions humaines (psychiatre de profession) et amateur des raisonnements menés sur la base de Fables (ayant fait publier par exemple dans sa jeunesse ses traductions en anglais de La Fontaine), est célèbre aujourd'hui pour la tentative de démonstration réalisée dans son poème La ruche mécontente, ou les coquins devenus honnêtes et dans les textes additionnels intégrés ensuite dans la Fable des abeilles : le vice conduit les sociétés humaines à l'efficacité collective. Le poème décrit en particulier des abeilles dans une ruche qui ont désiré supprimer toute l'abjection présente en son sein et finissent par se rendre compte qu’en procédant ainsi, elles ont ruiné leur société : rendues prospères par leurs vices, elles sont devenues pauvres en tentant de devenir vertueuses. Devenu célèbre par ses provocations (voir, l'incorporation de son Essai sur la charité et les écoles de charité dans la Fable) après une longue période d’indifférence à son endroit, Mandeville a bénéficié ensuite d'une étrange postérité car, même si sa vision noire de la nature humaine n’a jamais cessé d’être décriée à juste titre, il s'est trouvé rapidement considéré comme un «théoricien» dans le cadre de courants de pensée divers (Carrive, I980). Il présente notamment la particularité d’être appré-

I Cet article s'appuie sur l'ensemble des textes relevant de La Fable des abeilles. La Fable des abeilles, première partie (1705-1724) des éditions Vrin (2007) présente les écrits déjà inclus dans l'édition anonyme de La Fable des abeilles (17|4), La ruche mécontente, ou les coquins devenus honnêtes (1705), Recherches sur l'origine de la vertu morale (17|4), Remarques (17|4)), ainsi qu'un des deux essais ajoutés ensuite (Essai sur la charité et les écoles de charité (1723)) et un dossier (Défense du livre contre les accusations contenues dans la déclaration du grand jury du Middlesex (1724)). Le deuxième essai ajouté par Mandeville intitulé Recherche sur la nature de la société (1723) est traduit par Babel (1998). La Fable des abeilles, deuxième partie (1729), qui contient une «préface» et six célèbres «dialogues», est proposée par les éditions Vrin (1998). Tous les passages cités sont tirés de ces références pour la traduction en français. 
hendé depuis Hayek (1966) comme le premier grand théoricien de la formation d’un ordre spontané en précédant alors David Hume (Némo, 1988, Le Jallé, 2003) après avoir été considéré comme ayant fourni paradoxalement les outils logiques ayant servi de base à l'utilitarisme (avant Hume et Helvétius selon Bentham).

Si Mandeville est considéré avec autant d'intérêt, c'est sans doute car sa vision des fondements de la civilisation n’a jamais cessé de «troubler ». Derrière ses propos provocateurs, Mandeville s'est interrogé en effet avec causticité sur les mécanismes à l'origine de la formation de la superstructure morale et juridique que les hommes ont mis en place pour parvenir à «coopérer». Il a proposé dans ce cadre une première thèse, fort célèbre, suggérant que les hommes, grâce à leurs «défauts » (l'orgueil qui pousse à désirer être applaudi et la volonté de maximiser son utilité en particulier), sont parvenus à se réguler pour se comporter «comme il faut» et ainsi à «collaborer» entre eux dans un avantage soi-disant commun. Ce premier niveau de raisonnement, qui ne présentait en son temps rien de vraiment original, s'est avéré ensuite retourné par les Lumières (Hume et Helvétius au premier plan), puis par les utilitaristes². Mais ce qui fait son véritable intérêt est qu'il a développé aussi de façon sousjacente une seconde thèse bien plus féroce suggérant que ce processus d'autorégulation s'est fait en grande partie à l'instigation des «pires des hommes », ces derniers cherchant continuellement à se servir de la morale créée artificiellement pour pouvoir exploiter leur prochain et ayant pour cette raison participé grandement à son institution. Ce second aspect de la pensée mandevillienne, qui sera repris par Rousseau (la vision opposée qu'ils ont quant à la fonction de

2 Le principe que Mandeville défend est, pour les premiers tenants de l'utilitarisme (en notant qu'ils ignoraient l'influence chez lui des «plus méchants» dans le processus de régulation comportementale), celui de la maximisation du bonheur. L'auteur de la Fable des abeilles appelait en effet selon eux vices ce qui peut être tenu pour des vertus: «When Mandeville put forward his theory, that private vices are public benefits, he did not perceive that the erroneous application of the terms vice and virtue was the source of the confusion which enabled him to advocate a seeming contradiction. For if what is called virtue produce a diminution of happiness, and if vice, being the opposite of virtue, have a contrary effect, it is clear that virtue is the evil and vice the good, and that the principle which he advocates is merely the greatest-happiness principle under a cloud. If a private vice be on the whole instrumental in producing a result of felicity to the community, all that can be said is, that the vice has been christened by an erroneous and mistaken name». (Bentham-Bowring, 1834). 
la pitié ne devant pas induire en erreur), combattu par Hume (ce qui constitue peut-être la clé de l'opposition farouche entre ces deux derniers) et qui ne peut pas être passé sous silence chez ceux qui veulent comprendre le concept de «main invisible» chez Adam Smith, est pourtant comme placé à l'index. Sa prise en compte nécessite en effet de comprendre que, dans la civilisation qu'il décrit, la plupart des hommes ne cessent pas de se berner les uns les autres du fait de l'existence entre mandatés et mandataires d’asymétries informationnelles (ce qui fait de Mandeville une sorte de précédent à ceux qui examinent aujourd’hui la relation principal-agent) et qu'il raisonne sur cette base sur les mêmes sortes de fables que celles retenues aujourd'hui par les théoriciens des jeux. Cet article vise à partir de ce constat à présenter le rôle des «plus méchants » dans la théorie de l'évolution culturelle dressée par Mandeville pour préciser quels sont pour lui les fondements cachés de la formation de la civilisation.

\section{LA DISTINCTION DU «VIL»ET DU «NOBLE» COMME PRINCIPE DE RÉGULATION SOCIALE}

Pour apprécier le système de Mandeville, il est d’abord impératif de garder à l'esprit que le courant du pessimisme augustinien dont il relève avec par exemple La Rochefoucauld ${ }^{3}$, Esprit, Nicole... ne cessait pas au XVII ${ }^{\mathrm{e}}$ siècle sur un mode religieux ou moqueur (Lafond, 1990) d'expliquer que les hommes « déchus» pouvaient quand même se comporter d'eux-mêmes apparemment «comme il faut» en étant portés en vérité par un amour-propre «régulé », donc par la volonté de maximiser leur utilité à long terme et le désir d'être estimés pour cela, ainsi que le dessein d'exploiter leur prochain :

Ce que nous prenons pour des vertus n'est souvent qu'un assemblage d'intérêt que la fortune ou notre industrie savent arranger. (La Rochefoucauld, Maxime I, p. 75)

3 Pour créer leurs propres systèmes, les théoriciens français des Lumières tels qu'Helvétius se sont inspirés des dispositifs augustiniens basés sur l'amour-propre en leur retirant leur dimension culpabilisante (l'orgueil régulé et la recherche du maximum d'utilité n'étant plus dès lors présentés comme vicieux). C'est ainsi qu'Helvétius, pour qui l'intérêt est l'âme de l'amour-propre, rend hommage à La Rochefoucauld en regrettant que sa manière laisse penser que le vice est la source de la morale (Domenech, 1989). 
Ce que les hommes ont nommé amitié n’est qu'une société, qu'unménagement réciproques d'intérêts ${ }^{4}$, et qu'un échange de bons offices; ce n’est enfin qu'un commerce où l'amour-propre propose toujours quelque chose à gagner. (La Rochefoucauld, Maxime 83, p. 90)

Depuis que l'amour-propre s'est rendu le maître et le tyran de l'homme, il ne souffre en lui aucune vertu ni aucune action vertueuse qui ne lui soit utile, et qu'il les emploie toutes à faire réussir ses différentes prétentions [...] Ainsi, ils ne sacquittent ordinairement de tous ces devoirs que par le mouvement de l"amour propre et pour procurer l'exécution de ses desseins. (Esprit, La fausseté des vertus humaines, Préface)

Quoiqu'il n'y ait rien de si opposé à la charité qui rapporte tout à Dieu, que l'amour-propre qui rapporte tout à soi, il n'y a rien néanmoins de si semblable aux effets de la charité que ceux de l'amour-propre. Car il marche tellement par les mêmes voies, qu'on ne saurait presque mieux marquer celles où la charité nous doit porter, qu’en découvrant celles que prend un amour-propre éclairé, qui sait connaître ses vrais intérêts; et qui tend par raison à la fin qu'il se propose. (Nicole, De la charité et de l'amour-propre, p. 38I)

Bayle, qui peut être appréhendé comme un préalable «oublié » des théoriciens de la formation d'un ordre moral et juridique spontané, expliquait à la même époque sur un mode différent qu'une civilisation composée d'athées ne différerait pas sur le plan des actions civiles et morales d'une société de chrétiens grâce notamment au sens de l'honneur :

On voit à cette heure combien il est apparent qu'une société d'Athées pratiquerait les actions civiles et morales aussi bien que les pratiquent les autres sociétés, pourvu qu'elle fit sévèrement punir les crimes, et qu'elle attachât de l'honneur et de l'infamie à certaines choses... (Bayle, Pensées diverses écrites à un docteur de Sorbonne à l'occasion de la Comète qui parut au mois de décembre I680, Pensée I72, CLXXII, p. 48-52)

Imprégné par ces divers selfish systems of morals, Mandeville a tenté alors d'expliquer dans les textes associés à son célèbre poème comment les hommes ont pu créer de proche en proche une civilisation aussi noire que celle décrite par La Rochefoucauld et Esprit tout en s'avérant performante sur le plan de l'efficacité collective et a créé de cette façon une véritable théorie de l'évolution cultu- 
relle. L'auteur de la Fable des abeilles était motivé alors expressément en ce début du XVIII $^{\mathrm{e}}$ siècle par la volonté de se moquer des premières Lumières «sentimentales ${ }^{5} »$, en particulier Shaftesbury (I67I-I7I3) qui faisait de la «sympathie» (définie alors comme une sorte d’amour de l'humanité 6 ) le fondement de la tendance des hommes à collaborer entre eux et de cette façon de la civilisation, sachant qu'il raillait aussi le «sens du devoir» dont parlait Cicéron quand il se référait à la philosophie antique et à tous ceux qui s'en sont inspirés.

Pour construire sa théorie, Mandeville part de l'idée commune en son temps et qui sera reprise par l'utilitarisme selon laquelle les gens apprennent par leur éducation et leur formation à réguler leur orgueil pour parvenir à se comporter «comme il faut» dans l'intérêt collectif. Dans ses Recherches sur l'origine de la vertu morale que l'on peut appréhender comme le texte clé pour aborder sa logique, l'homme est présenté ainsi de prime abord comme un animal d'un égoïsme et d'une obstination, aussi bien que d'une ruse extraordinaires, mais qui peut dompter ses appétits, sachant qu'il est impossible par la violence seule de le rendre traitable. Il y explique que les gens à leur état naturel (avant la civilisation) aimaient trop le présent par rapport au futur et avaient ainsi une tendance à la non coopération systématique car ils n'étaient pas dotés en plus d’un orgueil éclairé : les «hommes sauvages » n'étaient pas vraiment capables de collaborer entre eux par intérêt car ils étaient obnubilés par la volonté de maximiser leur utilité à court terme. Les individus ont senti alors que le principal obstacle à la coopération dans leurs interactions était cette trop grande préférence pour le présent si bien

5 Sur les deux camps en présence au début du $\times$ VIII ${ }^{e}$ siècle, voir Hutcheson (I728), grand détracteur de Mandeville du vivant de ce dernier, p.22: «ll y a deux opinions à ce sujet entièrement opposées: l'une, celle des anciens épicuriens [...] qui est ressuscitée par Hobbes, La Rochefoucauld [...] que tous les désirs humains [...] sont réductibles à l'amour de soi, ou désir de bonheur privé [...] L'autre opinion est celle-ci, que nous n'avons pas seulement l'amour de soi, mais aussi des affections bienveillantes à l'égard d'autrui, à des degrés divers, qui nous font désirer leur bonheur comme fin ultime».

6 Hume, qui se situe entre le camp «égoïste» allant de Hobbes à Mandeville et celui «bienveillant» de Shaftesbury et Hutcheson en considérant que les hommes ne sont pas dotés d'un amour pour l'humanité, mais d'une générosité limitée, a proposé ensuite une conception plus «scientifique» de la sympathie (prise cette fois comme principe de transmission des passions d'une personne à l'autre). 
qu'ils ont créé spontanément une superstructure morale et juridique poussant à dresser des collaborations durables entre eux.

Pour Mandeville, les gens en se servant des «lawgivers and other wise men » (qui sont l'émanation des hommes eux-mêmes en précisant aussitôt qu'ils sont poussés en grande partie par les «plus méchants» pour signer l'existence d’un second niveau de raisonnement) ont décomposé ainsi dans la nuit des temps l'humanité en deux sortes d'individus pour rendre les hommes utiles les uns aux autres aussi bien que dociles. Ces deux types sont d'une part les gens «vils» (abject, low minded people) uniquement préoccupés par la volonté de maximiser leur utilité à court terme et qui vont servir de repoussoir, d'autre part les gens «nobles » (high-spirited creatures) $)^{7}$ capables de freiner leur envie de maximiser leur utilité à court terme dans les domaines considérés comme bien en vertu de la superstructure morale mise justement en place par les «lawgivers and other wise men »:

L’une [classe] consistait en gens vils [souligné par nous], aux préoccupations basses qui, toujours à la recherche de jouissances immédiates, étaient totalement incapables d'abnégation, et qui, dénués de considération pour le bien d'autrui, n'avaient pas de but plus noble que leur avantage particulier. Ces genslà, esclaves de la volupté, s'abandonnaient sans résistance à tous les désirs grossiers et ne faisaient usage de leurs facultés rationnelles que pour accroitre leur plaisir sensuel. Ces hommes-là sont le rebut de leur espèce.

Mais l'autre classe était faite de créatures nobles [souligné par nous], à l'esprit élevé, qui, exemptes d'un égoïsme sordide, voyaient dans la culture de l'esprit la plus belle possession et qui, connaissant leur vraie valeur, ne trouvaient de délices qu'à orner la partie d'eux-mêmes en quoi consiste leur excellence. Ces gens-là, méprisant tout ce qu'ils avaient en commun avec les créatures dépourvues de raison, s'opposaient avec l'aide de la raison à leurs inclinaisons les plus violentes, et se faisant à eux-mêmes une guerre continuelle, ils ne visaient à rien de moins qu'à assurer le bien public et à conquérir leur propre passion [...]

7 La distinction entre abject, low minded people et high-spirited creatures a été reprise (Carrive, 1980) dans le système de l'amour-propre de Rousseau (1755) sous la forme d'une opposition entre «canailles» et «honnêtes gens». Rousseau, même s'il repousse l'idée d'une inclinaison naturelle des hommes pour le mal et inverse le rôle conféré à la pitié dans la Fable des abeilles, peut être considéré de ce point de vue comme un descendant critique de Mandeville (voir Letter to the authors of Edinburgh Review (1755) d'Adam Smith qui est l'un des premiers à avoir compris la ressemblance paradoxale entre les systèmes de Rousseau et Mandeville). 
Ce sont ces derniers qu'ils [les «législateurs et autres sages»] ont appelés les véritables représentants de leur sublime espèce, ceux qui surpassent davantage en dignité la première classe que celle-ci était elle-même au dessus des bêtes brutes. (Mandeville, Recherches sur l'origine de la vertu morale, p. 45)

Mandeville, chez qui la distinction artificielle entre vils et nobles est à la base de la formation de la morale (la façon dont l'homme sauvage a été dompté), introduit de cette façon l'orgueil régulé qui rend les gens prêts à «endurer mille désagréments et supporter mille fatigues pour avoir le plaisir de se compter dans la seconde classe [noble] et par conséquent de s'approprier toutes les excellences qu'ils lui ont entendu attribuer ». Ce système de valeurs conventionnel, qui vise à donner dans certains domaines de la satisfaction à ceux qui adoptent une retenue, a été mis en place par les hommes parce qu'il leur permettait apparemment de collaborer entre eux dans un avantage commun (une «foule d'ennuis » s'abattant sur eux s'ils ne jouaient pas le jeu du respect de la coopération) :

Une fois posés ces fondements de la politique [l'opposition entre nobles et vils], il était impossible que l'homme restât longtemps sans être civilisé. Car même ceux qui ne travaillaient qu'à satisfaire leurs appétits, se trouvant continuellement contrariés par d'autres personnes semblablement disposées, n’ont pas pu ne pas s'apercevoir que chaque fois qu'ils réprimaient leur inclinaison, ou simplement mettaient plus de prudence à la suivre, ils s'épargnaient une foule d'ennuis, et évitaient souvent beaucoup de ces malheurs qui accompagnent en général la poursuite trop avide de plaisirs [souligné par nous].

Premièrement, ils recevraient le même profit que les autres des actions accomplies pour l'ensemble de la société, et par conséquent ils ne pouvaient s'empêcher d'en savoir gré à ceux de la classe supérieure qui les avaient accomplies. Deuxièmement, plus ils étaient acharnés à chercher leur propre avantage sans égard pour autrui, plus ils se persuadaient qu'ils navaient pas de plus grands adversaires que ceux qui leur ressemblaient le plus. (Mandeville, Recherches sur l'origine de la vertu morale, p. 46-47)

À première vue, les gens ont construit ainsi spontanément une civilisation telle que les louanges qu'ils attirent en adoptant une attitude favorable au bien public vient augmenter leur satisfaction, ce qui pousse à se comporter de façon noble plutôt que vile, donc «comme il faut » (l'espèce humaine se construisant passionnellement en fonction de ses intérêts) : 
Plus nous examinerons de près la nature humaine, plus nous nous convaincrons que les vertus morales sont la progéniture politique que la flatterie et l'orgueil ont engendré à eux deux. (Mandeville, Recherches sur l'origine de la vertu morale, p. 49)

Il n'y a aucun de ces bons offices ou devoirs, envers autrui ou envers nousmêmes, dont parlait Cicéron, ni de ces cas de bonté, d'humanité ou d'une autre vertu sociale auxquels faisait allusion milord Shaftesbury, qu'un homme de bon sens $[\ldots]$ ne puisse apprendre à pratiquer sans être dirigé par un meilleur principe que la vaine gloire. (Mandeville, Cléomène $2^{\mathrm{e}}$ dialogue, p. 6I)

C'est pour Mandeville une caractéristique clé des hommes, le fait qu'ils aient de l'amour-propre (donc qu'ils aient peur de n'être pas à la hauteur de leurs espérances et soient ainsi obsédés par le désir de faire leur preuve) qui a permis de tirer partie de cette distinction artificielle entre «vil » et « noble » :

J'imagine que, pour accroitre le soin que prennent les créatures de se préserver elles-mêmes, la nature leur a donné un instinct, par lequel tout individu s'estime au dessus de sa valeur réelle; cet instinct [...] semble s'accompagner d'une défiance de soi, qui vient de la conscience ou du moins de la crainte, que nous avons, que peut-être nous nous estimons excessivement. C'est ceci qui nous fait tant aimer l'approbation, l'affection et l'assentiment des autres, parce que cela nous renforce et nous confirme dans la bonne opinion que nous avons de nous-mêmes. (Mandeville, Cléomène $3^{\mathrm{e}}$ dialogue, p. II3)

Si donc on veut donner de cette récompense qu'est la gloire la définition la plus large, tout ce qu'on peut en dire, c'est qu'elle consiste en une félicité suprême dont l'amour-propre fait jouir celui qui, conscient d'avoir accompli une noble action, pense aux applaudissements qu'il attend des autres. (Recherches sur l'origine de la vertu morale p. 5I)

L'auteur de la Fable des abeilles précise que les hommes auraient dressé aussi de proche en proche un ordre juridique adapté pour appuyer ${ }^{8}$ l'outil que constitue cette régulation morale :

8 Hume (1740, 175I), dans sa propre théorie de la formation d'un ordre spontané (au sens non hayékien du terme d'un constructivisme autorégulé (Le Jallé, 2003, 2005), postulera que les gens ont créé leurs vertus artificielles avant d'instaurer un ordre juridique coercitif. Par exemple, si les hommes dans leurs relations économiques ont pu se mettre à rendre service dans l'attente d'un retour éloigné dans le temps (voir la «fable des paysans» dans Hume, 1740) dans une petite société sans «gouvernement» au début de la civilisation, c'est pour lui parce 
Si vous voulez vous donner cette peine, vous découvrirez que le nombre de clauses $[\ldots]$ nécessaires pour bien gouverner une grande ville riche sont quelque chose de plus prodigieux qu'on ne saurait l'imaginer, et que pourtant il n'y en a pas une qui ne vise au même but, brider, restreindre et déjouer les passions excessives et les nuisibles faiblesses des hommes [...] De tout ce que j'ai mentionné, il y a en bien peu qui soient l'ouvrage d'un seul homme ou d'une seule génération, la plus grande partie est le produit, l'œuvre commune de plusieurs siècles [...] La sagesse dont je vous parle n'est pas le fruit d'une belle intelligence ou d'une pensée profonde mais d'un jugement sain et réfléchi, acquis par une longue expérience des affaires et par des observations nombreuses. (Mandeville, Cléomène 6 dialogue, p. 263-264)

\section{LA RAISON CACHÉE DE LA FORMATION DE L'ORDRE MORAL ET JURIDIQUE}

Pouvoir exploiter son prochain

S'il s'était contenté d'expliquer que les membres de l'espèce humaine se sont régulés via leur orgueil pour parvenir à se comporter «comme il faut» et ainsi à collaborer entre eux, il serait difficile d'affirmer que Mandeville présente une vraie noirceur. En effet, dès la parution de la Fable, les commentateurs comme Charles-Irénée Castel de Saint Pierre, avant que cette idée ne soit reprise par les philosophes des Lumières ayant servi de «boite à outils » à l'utilitarisme (Hume et Helvétius en particulier), ont noté que les défauts dont il parle au premier plan (l'orgueil et la volonté de maximiser son utilité) peuvent être présentés comme des qualités9 puisqu'ils conduisent au bien collectif. Mais Mandeville ne s'est pas borné à dire que les hommes ont institué d'eux-mêmes un système de valeurs fait pour freiner leur envie de maximiser leur utilité à court terme (créant ainsi une civilisation efficace). Il a ajouté un second niveau d'explication en manifestant que le processus d'édification de la superstructure morale et politique a été mis en place en grande partie à l'instigation des «plus

qu'ils se sont autorégulés en commun en créant de proche en proche un sens de l'honneur conscientisé relatif au respect de la parole donnée à autrui (les signes ou sons signifiant «je promets» émergeant conventionnellement de façon spontanée).

9 Voir Hume dans De la dignité ou de la bassesse de la nature humaine, I74I, p. 225. 
méchants » (the very worst of them) dans une vue d'exploitation de son prochain. Les «lawgivers and other wise men » sont poussés en effet selon Mandeville par les pires des hommes parce que ces derniers tirent perpétuellement entre eux avantage de la distinction «vil»/ «noble» et cherchent ainsi continuellement à se servir de la morale pour assurer leur réussite ${ }^{10}$ :

Le principal but, donc que se sont proposés les « lawgivers and other wise men» qui ont travaillé a établir la société, a été de faire croire aux gens qu’ils avaient à gouverner qu'il était plus avantageux pour chacun de dompter ses appétits que de leur donner libre cours et qu'il valait bien mieux pour lui veiller à l'intérêt public qu'à ce que lui semblerait son intérêt particulier [souligné par nous]. Cela a toujours été une tâche bien difficile, aussi n'y a-t-il pas de bel esprit ou d'orateur qu'on n'ait cherché à enrôler à son service; et les moralistes et les philosophes de tous les temps ont employé toute leur adresse à prouver une opinion aussi utile [...] Il est donc dans l'intérêt des plus méchants d'entre eux [des hommes] plus que d'aucun autre de prôner le dévouement au bien public, qui leur permettrait de recueillir le fruit du travail et de l'abnégation des autres, et en même temps, d'être plus tranquilles pour laisser le cours libre à leurs appétits [souligné par nous]; ils s'accordèrent pour appeler VICE tout ce que, sans égard pour l'intérêt public, l'homme commet pour satisfaire un de ses appétits si dans cette action on peut observer la plus légère possibilité qu'elle nuise à un membre de la société ou qu’elle rende son auteur jamais moins utile aux autres. Ils s'accordèrent pour donner le nom de VERTU à toute action dans laquelle l'homme, allant contre l'impulsion naturelle, s'efforce de faire du bien aux autres, ou de vaincre ses passions par une ambition rationnelle d'être un homme de bien. (Mandeville, Recherches sur l'origine de la vertu morale, p. 43-47)

Mandeville donne tellement d'importance à ce fondement caché de la superstructure morale et politique existant dans la civilisation que c'est sans nul doute pour s'expliquer sur ce point qu'il a écrit son célèbre poème La ruche mécontente, ou les coquins devenus honnêtes. Il imagine en effet dans ce cadre l'existence d'un monde

I0 Faisant un parallèle avec la manière dont le langage s'est mis en place conventionnellement, Mandeville a construit un modèle représentant le processus culturel et évolutif de mise en place des valeurs morales (voir le sens de l'honneur), ainsi que du cadre juridique adapté nécessaire pour que les hommes puissent collaborer entre eux. Les «économistes» des conventions contemporains préfèrent trouver leurs précédents (Postel-Sobel, 2006, Salais, 2009) chez Lewis (1969) (Le Jallé, 2009) et plus loin encore chez Hume (voir le traitement de la fable des rameurs dans Hume, 1740, 175I). Ce dernier affirmait que le concept de convention utilisé par lui était déjà présent chez Grotius dont la proximité avec Pufendorf en la matière est souvent rapportée (Le Jallé, 2005), cette donnée étant déjà présente chez Stewart (1828). 
artificiellement moral où tous les hommes-abeilles, sauf une minorité (surtout les plus pauvres d'entre eux), ne sont qu'intéressés et désireux d'être applaudis quand ils donnent l'impression d’agir dans le sens du bien collectif (par exemple pour un médecin de bien soigner, pour un avocat de défendre au mieux, pour un Grand au pouvoir d'être un homme d'État, pour un juge d’agir dans le sens de l'équité, pour un employeur de faire des affaires sans faire d'ententes avec d'autres pour exploiter ses ouvriers et sans mentir sur la qualité des biens qu'il vend, pour un soldat de se battre avec courage...). Le paradoxe que met en avant Mandeville à travers nombre d'exemples est alors qu'un tel dispositif moral est ce qu'il y a de mieux pour que les pires individus parviennent à s'enrichir le plus possible parce qu'il existe des asymétries informationnelles dans les relations entre gens passant l'équivalent de contrats en ce qui concerne le caractère adéquat d'un service rendu ${ }^{11}$.

Par exemple, dans son poème, nombre de «médecins » et d'« apothicaires et autres », présentés comme à la fois intéressés et soucieux de leur réputation, paraissent tenir à la santé de leurs patients sachant qu'au fond ils n’en ont rien à faire. Ils dressent alors entre eux des coalitions d'une extrême solidité car elles reposent sur une vérité cachée (ils trompent leur clientèle) dont la révélation viendrait ruiner leur réputation:

Exemple I (jeu des médecins et apothicaires):

Les médecins prisaient la gloire et l'argent plus que la santé du malade dépérissant ou que leur propre science. Le plus grand nombre s'appliquait, non aux règles de l'art, mais à un air grave et pensif, et une mine ${ }^{12}$ soucieuse pour se concilier la faveur des apothicaires, les éloges des sages-femmes, des prêtres et de tous ceux qui officiaient à la naissance ou aux funérailles; à supporter patiemment la tribu braillard [...] avec un sourire cérémonieux et un aimable compliment, à faire la cour à toute la famille; et, ce qui est encore le plus pénible, à souffrir l'insolence des garde-malades. (Mandeville, Le Poème, p. 3I)

II Les agents (mandatés) disposent dans la Fable d'avantages informationnels sur les principaux (mandataires) concernant d'une part leurs qualités (la sélection adverse), d'autre part leur niveau d'effort et le caractère approprié des efforts qu'ils mettent en œuvre (le risque moral). Les mandataires roulent aussi les mandatés.

12 «Dans toutes les professions chacun affecte une mine et un extérieur pour paraître ce qu'il veut qu'on le croie. Ainsi on peut dire que le monde n'est composé que de mines.» (La Rochefoucauld, Maxime 256, p. 123) 
Mandeville considère ainsi que la coopération entre ces «hypocrites » peut s'installer efficacement car elle s'inscrit dans un cadre moral, le raisonnement produit par lui renvoyant à une «discussion» sur le moyen de collaborer dans un jeu du type suivant (ressemblant à un dilemme du prisonnier) :

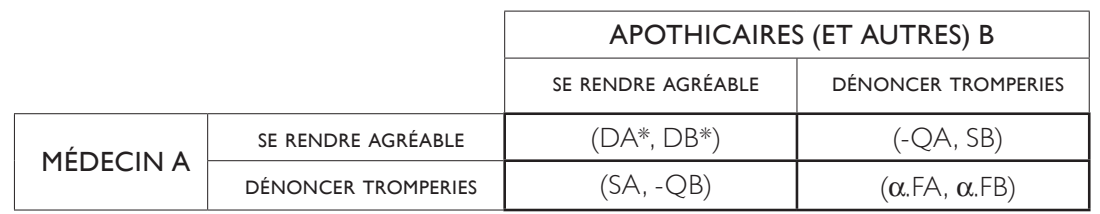

avec:

DA, DB: gain pour A (resp.pour B) de l'entente

SA, SB: produit pour A (resp.pour B) de la dénonciation de A (resp.de B)

-QA, -QB: préjudice pour A (resp.pour B) en cas de défection de $B$ (resp.de $A$ )

FA, FB: gain de $A$ (resp.de $B$ ) si les hommes ne dressent pas une entente (avec $\alpha<1$ très nettement)

et: $\mathrm{SA}>\mathrm{DA}>\mathrm{FA}>-\mathrm{QA}$ et $\mathrm{SB}>\mathrm{DB}>\mathrm{FB}>-\mathrm{QB}$

Exemple numérique:

\begin{tabular}{|c|c|c|c|}
\cline { 3 - 3 } \multicolumn{2}{c|}{} & \multicolumn{2}{c|}{ APOTHICAIRES (ET AUTRES) B } \\
\cline { 2 - 4 } \multicolumn{2}{c|}{} & SE RENDRE AGRÉABLE & DÉNONCER TROMPERIES \\
\hline \multirow{2}{*}{ MÉDECIN A } & SE RENDRE AGRÉABLE & $\left(8 \mathrm{~A}^{*}, 8 \mathrm{~B}^{*}\right)$ & $(-2 \mathrm{~A}, 10 \mathrm{~B})$ \\
\cline { 2 - 4 } & DÉNONCER TROMPERIES & $($ IOA, -2B $)$ & $(\alpha .2 \mathrm{~A}, \alpha .2 \mathrm{~B})$ \\
\hline
\end{tabular}

Dans le langage de la théorie des jeux ${ }^{13}$, la collaboration durable entre ces médecins et apothicaires se met en place ici en effet parce que chacun d'eux sait combien il est mutuellement fort couteux de trahir l'autre (ici de dénoncer aux tiers la tromperie) quand on se met à se rendre des services dans de telles situations (r étant le taux d’actualisation):

13 Ceux qui voient en David Hume le père du système philosophique en relation avec la théorie des jeux (voir Binmore, 2005, 2007, 2009 en particulier) devraient se tourner aussi vers Mandeville et ceux qui l'ont inspiré (La Rochefoucauld, Esprit, Nicole...). Afin d'éviter toute surinterprétation, il est important ici d'apprécier que Mandeville ne manifestait en rien le souci de formaliser mathématiquement son raisonnement ou d'utiliser des exemples chiffrés. Voir Dockès (2008) pour l'examen du rapport de Thomas Hobbes au DP indéfiniment répété. 


$$
\begin{aligned}
& \quad 8 A+8 A /(\mathrm{I}+\mathrm{r})+8 \mathrm{~A} /(\mathrm{I}+\mathrm{r})^{2}+8 \mathrm{~A} /(\mathrm{I}+\mathrm{r})^{3}+(\ldots)>\mathrm{IO} A+\alpha \cdot 2 A /(\mathrm{I}+\mathrm{r})+\alpha \cdot 2 A /(\mathrm{I}+\mathrm{r})^{2} \\
& +\alpha \cdot 2 A /(\mathrm{I}+\mathrm{r})^{3}+(\ldots) \\
& \quad 8 \mathrm{~B}+8 \mathrm{~B} /(\mathrm{I}+\mathrm{r})+8 \mathrm{~B} /(\mathrm{I}+\mathrm{r})^{2}+8 \mathrm{~B} /(\mathrm{I}+\mathrm{r})^{3}+(\ldots)>\mathrm{IOB}+\alpha \cdot 2 \mathrm{~B} /(\mathrm{I}+\mathrm{r})+\alpha \cdot 2 \mathrm{~B} /(\mathrm{I}+\mathrm{r})^{2} \\
& +\alpha .2 \mathrm{~B} /(\mathrm{I}+\mathrm{r})^{3}+(\ldots) \\
& \text { avec: } \alpha<\text { I très nettement car afficher aux spectateurs quand on a commencé une telle collaboration } \\
& \text { que l'on les trompe ses «clients » revient à s'exclure de la possibilité de gagner ensuite beaucoup d'ar- } \\
& \text { gent avec tous les autres clients potentiels. }
\end{aligned}
$$

Si ces médecins et apothicaires ont la possibilité de gagner le plus d’argent possible, c'est ainsi parce qu'il existe une superstructure morale, leurs clients croyant vraiment qu'ils tentent de les soigner au mieux alors qu'ils ne s'intéressent en fait qu'à la richesse qu'ils vont pouvoir tirer de leurs activités.

Idem, Mandeville décrit des avocats qui, faussement soucieux du sort de leurs clients, dressent entre eux des ententes secrètes (pour faire prolonger les audiences et augmenter ainsi leurs revenus) fort solides car la nature de leur coopération ne peut pas être avouée :

Exemple 2 (jeu des avocats):

Les avocats, dont l'art avait pour fondement de susciter des dissensions et de couper le droit en quatre [...] retardaient délibérément les audiences pour toucher de nouveaux honoraires. (Mandeville, Le Poème, p. 30-3I)

\begin{tabular}{|c|c|c|c|}
\cline { 3 - 4 } \multicolumn{2}{c|}{} & \multicolumn{2}{c|}{ AVOCAT B } \\
\cline { 3 - 4 } \multicolumn{2}{c|}{} & EN FAIRE TROP & DÉNONCER TROMPERIES \\
\hline \multirow{3}{*}{ AVOCAT A } & EN FAIRE TROP & $\left(8 A^{*}, 8 B^{*}\right)$ & $(-2 \mathrm{~A}$, IOB $)$ \\
\cline { 2 - 4 } & DÉNONCER TROMPERIES & $(\mathrm{IOA},-2 \mathrm{~B})$ & $(\alpha .2 \mathrm{~A}, \alpha .2 \mathrm{~B})$ \\
\hline
\end{tabular}

Dans un autre jeu de même nature, Mandeville décrit la relation entre des juges (soucieux de passer pour impartiaux) et des puissants (voulant passer pour de bons gouvernants) qui se rendent continuellement des services intéressés au détriment des plus pauvres, la force de la coopération étant assurée par la nécessité mutuelle de cacher au public le fait qu'ils le trompent: 
Exemple 3 (jeu de la connivence entre justice et puissants):

La justice elle-même $[\ldots]$ sa main gauche, qui doit tenir la balance, la lâchait souvent, subornée à prix d’or... le glaive dont elle est armée n’arrêtait que les pauvres et les gens réduits à l'extrémité [...] pour des crimes qui ne méritaient pas ce sort uniquement afin de protéger les riches et les grands. (Mandeville, Le Poème, p. 32-33)

\begin{tabular}{|c|c|c|c|}
\hline & & \multicolumn{2}{|c|}{ PUISSANT B } \\
\hline & & ARRANGEMENT & DÉNONCER TROMPERIES \\
\hline \multirow{2}{*}{ JUGE A } & ARRANGEMENT & $\left(8 A^{*}, 8 B^{*}\right)$ & $(-2 A, 10 B)$ \\
\hline & DÉNONCER TROMPERIES & $(10 A,-2 B)$ & $(\alpha .2 \mathrm{~A}, \alpha .2 \mathrm{~B})$ \\
\hline
\end{tabular}

Dans un autre exemple, Mandeville décrit les ententes entre ceux qui emploient d'autres hommes en exploitant ces derniers (par exemple, ils s'arrangent entre eux pour ne pas les payer assez), tout en étant désireux se faire passer pour des entrepreneurs honnêtes :

Exemple 4 (jeu de l'entente entre employeurs):

Et tous ceux qui, ennemis du simple travail, se débrouillent pour détourner à leur profit le labeur de leur prochain, brave homme sans défiance (Mandeville, Le Poème, p. 30)

\begin{tabular}{|c|c|c|c|}
\cline { 3 - 4 } \multicolumn{2}{c|}{} & \multicolumn{2}{c|}{ EMPLOYEUR B } \\
\cline { 3 - 4 } \multicolumn{2}{c|}{} & ARRANGEMENT & DÉNONCER TROMPERIES \\
\hline \multirow{2}{*}{ EMPLOYEUR A } & ARRANGEMENT & $\left(8 A^{*}, 8 B^{*}\right)$ & $(-2 \mathrm{~A}, 10 \mathrm{~B})$ \\
\cline { 2 - 4 } & DÉNONCER TROMPERIES & $(10 \mathrm{~A},-2 \mathrm{~B})$ & $(\alpha .2 \mathrm{~A}, \alpha .2 \mathrm{~B})$ \\
\hline
\end{tabular}

Mandeville présente de même les ententes solides (à ne surtout pas révéler car la réputation des personnes concernées serait ruinée si l'un d'eux parlait) entre des gens faisant des affaires entre eux en trichant par exemple sur la qualité de ce qu'ils vendent: 
Exemple 5 (jeu des carotteurs):

Les immondices même que dans les rues on vendait comme engrais, L'acheteur les trouvait bien souvent adultérées par un quart de pierres [...] sans valeur. Mais carotteur n'avait guère le droit de murmurer, lui qui vendait à l'autre du sel au prix du beurre. (Mandeville, Le Poème, p. 32)

\begin{tabular}{|c|c|c|c|}
\cline { 3 - 4 } \multicolumn{2}{c|}{} & \multicolumn{2}{c|}{ CAROTTEUR B } \\
\cline { 2 - 4 } \multicolumn{2}{c|}{} & SE TAIRE & DÉNONCER TROMPERIES \\
\hline \multirow{2}{*}{ CAROTTEUR A } & SE TAIRE & $\left(8 \mathrm{~A}^{*}, 8 \mathrm{~B}^{*}\right)$ & $(-2 \mathrm{~A}, 1 \mathrm{~B})$ \\
\cline { 2 - 4 } & DÉNONCER TROMPERIES & $($ IOA, -2B $)$ & $(\alpha .2 \mathrm{~A}, \alpha .2 \mathrm{~B})$ \\
\hline
\end{tabular}

De cette façon, nombre de médecins ou apothicaires (qui veulent laisser paraître qu'ils sont d'abord soucieux de bien soigner), d’avocats (de bien défendre), de juges (d'être impartiaux), de puissants (d'être des hommes d'État), d'employeurs (de gagner leur argent honnêtement), d'hommes d’affaires (de réaliser des affaires sans tricher)... se servent du sens du bien collectif véhiculé par les «lawgivers and other wise men » pour mieux tromper les autres hommes et augmenter leurs richesses. Si la superstructure n'existait pas, donc si les gens qui établissent des contrats avec eux ne croyaient pas que ces individus travaillent «comme il faut» pour eux, ces «scélérats » ne gagneraient pas autant d'argent. Pour Mandeville, la superstructure morale et politique ne s'est pas ainsi seulement mise en place parce que les membres de l'espèce humaine applaudissent les gens qui paraissent agir dans le sens du bien collectif. C'est aussi parce qu'elle permet aux «plus méchants » de s'enrichir continuellement en exploitant son prochain et de s'en sortir toujours mieux que les autres. Puisque les vertus morales autres que naturelles et le cadre juridique associé doivent ainsi en partie leur existence à l'amour des commodités de l'existence de nombre d'hommes et à la nécessité d'« endormir » les consciences de ceux avec qui l'on contracte, il est ainsi ridicule selon lui de considérer comme Shaftesbury que la civilisation est fondée sur la sympathie au sens d'un amour de l'humanité. Il est important de noter que, chez Mandeville, les médecins, avocats, juges, puissants, employeurs, hommes d’affaires... ne relèvent pas tous des «plus méchants», ce qui participe à la crédulité générale de la «clientèle» des abeilles scélérates. 


\section{DE LA NÉCESSITÉ DE NE PAS LAISSER TOUTES LES SORTES DE MANDATÉS EXPLOITER LEURS MANDATAIRES}

Le sort funeste réservé aux pauvres faits soldats et ouvriers

Le système mandevillien examine ainsi la mise sur pied d'une civilisation où nombre de mandatés trompent leurs mandataires et vice versa du fait de l'existence d'asymétries informationnelles entre eux. Pour Mandeville, les hommes de troupe et les ouvriers disposent a priori des mêmes possibilités que tous les autres mandatés (avocats, médecins, employeurs...) s'ils ont reçu suffisamment d'éducation pour savoir déroger «hypocritement » aux règles morales. L'argumentation précise qu'il donne en la matière à propos des comportements des soldats permet de comprendre en profondeur sa pensée. Dans une première mise en situation, Mandeville explique en effet d'abord que chacun des troupiers d'une même armée donnée serait enclin pendant la bataille, s’il n'était pas poussé par l'orgueil régulé (l'honneur tiré du courage physique ici), à vouloir maximiser son utilité à court terme (fuir) plutôt que maximiser son utilité à long terme (se battre au mieux avec les autres), sachant que le risque de se faire tuer devient fort grand si l'on reste peu nombreux à se battre et que la collaboration conduit à l'optimum collectif (les hommes augmentant leur chance de victoire rapide et de survie en cas d'engagement maximal de toute l'armée) :

Les soldats, forcés de se battre, s'ils survivaient, y trouvaient de l'honneur. D’autres, pourtant, voulant éviter la sanglante mêlée, se faisaient emporter un membre en s'enfuyant. (Mandeville, Le Poème, p. 3I)

L'honneur militaire créé par l'entremise des «lawgivers and other wise men» (parce qu'il est bon pour le bien collectif) sert donc clairement de Léviathan moral pour les obliger à contenir leur envie de fuir (de tout faire pour tenter de sauver sa vie à court terme), la coercition (instituer une forte discipline) s'imposant de façon additionnelle: le fait que les hommes applaudissent les gens (ici les soldats) qui agissent dans le sens du bien collectif a bien créé autrement dit un sens de l'honneur artificiel leur permettant de se battre avec le plus d'efficacité possible pendant une bataille. Mais Mandeville n’arrête pas là son raisonnement : il explique combien il est question en fait dans ce jeu de 
pauvres qui sont destinés à servir de chair à canon potentielle au profit de leur communauté et qui s'avèreront ainsi peut-être sacrifiés à terme au profit de tous (ce qui revient encore une fois à moquer le positionnement de Shaftesbury car une civilisation prête à envoyer des jeunes hommes se faire massacrer ne peut pas être considérée comme régie par l'amour de l'humanité). Mandeville, qui fait clairement la différence dans les jeux guerriers entre officiers et simples soldats, postule autrement dit que l'on fait croire ici à des pauvres transformés en combattants qu'ils se rapprochent de la «noblesse» comportementale en agissant avec courage alors qu'ils se font en fait posséder dans l'intérêt de la société et surtout des puissants qui les utilisent sans états d'âme:

Mais les moyens qu'on prend pour exciter l'orgueil des hommes, et les faire prisonniers par là, ne sont nulle part plus grossièrement éclatants que dans la manière dont on traite les simples soldats dont il faut travailler la vanité, puisqu'ils sont si nombreux, au meilleur marché possible [...] Le plus rude tissu de laine [...] lui plait car il imite le teint d'une couleur écarlate [de l'officier] ne faut-il pas s'étonner qu'il y ait de pauvres misérables assez sots pour être fiers de s'entendre appeler Messieurs les soldats. (Mandeville, Remarque R, p. 167-168)

Mandeville ajoute alors que les soldats de troupe ne sont pas différents des autres mandatés et qu'ils peuvent, comme les avocats, les médecins... «abuser» de la situation pour chercher à s'en sortir au mieux et à s'enrichir. Pour exprimer cette situation, il manifeste dans le poème, tel un précédent caustique à Axelrod ${ }^{14}$, que, tandis que nombre de troupiers dont le sens de l'honneur militaire a été attisé collaborent et se retrouvent à servir de chair à canons, d’autres profitent de la situation en sarrangeant entre eux, même quand ils sont ennemis, pour ne pas se retrouver au feu et tirer un avantage économique du courage naïf des autres :

14 Voir le système «vivre et laisser vivre» durant la lre guerre mondiale: les soldats ennemis (français et allemands par exemple) faisaient croire à leurs commandements respectifs qu'ils se battaient alors qu'ils évitaient les confrontations brutales en se comportant donnant-donnant les uns vis-à-vis des autres (Axelrod, 1984). 
Exemple 6 (second jeu des soldats pendant la bataille):

Parfois un valeureux général cherchait l'ennemi [...] un autre, soudoyé pour cela, le laissait passer. Les uns s'aventuraient toujours au plus fort du combat [.... Ils perdaient tantôt une jambe, tantôt un bras, jusqu'à ce que tout à fait invalides et mis au rencart, ils vécurent de leur demi-solde. Tandis que d’autres, sans jamais entrer en jeu, restaient à l'arrière, et recevaient double paie. (Mandeville, Le Poème, p. 3I-32)

\begin{tabular}{|c|c|c|c|}
\cline { 3 - 3 } \multicolumn{2}{c|}{} & \multicolumn{2}{c|}{ SOLDAT ADVERSAIRE B } \\
\cline { 2 - 4 } \multicolumn{2}{c|}{} & ÉVITER LE COMBAT & DÉNONCER TROMPERIES \\
\hline \multirow{3}{*}{ SOLDAT A } & ÉVITER LE COMBAT & $\left(8 \mathrm{~A}^{*}, 8 \mathrm{~B}^{*}\right)$ & $(-2 \mathrm{~A}, 10 \mathrm{~B})$ \\
\cline { 2 - 4 } & DÉNONCER TROMPERIES & $(10 \mathrm{~A},-2 \mathrm{~B})$ & $(\alpha .2 \mathrm{~A}, \alpha .2 \mathrm{~B})$ \\
\hline
\end{tabular}

Ces hommes de troupe donnant-donnant se servent bien ainsi de la morale (risquant d'être fusillés s'ils se font prendre à tricher) pour s'en sortir en trompant les autres tout en désirant être applaudis pour un comportement « comme il faut». Mandeville dresse alors sur cette base un parallèle entre les hommes de troupe et les ouvriers pauvres: comme les soldats, les travailleurs salariés ont tendance à se faire exploiter par des employeurs qui abusent d'eux pour améliorer leur propre sort. Nombre d'entre eux, surtout quand ils sont élevés dans les écoles de charité car elles viennent donner un esprit frondeur à ceux qui en profitent (voir l'« esprit» des serviteurs de chez Molière), n’hésitent pas alors à s'entendre entre eux pour ne pas faire ce qui est demandé par leurs employeurs et refuser «insidieusement» tout travail par trop pénible.

L'Essai sur la charité et les écoles de charité, celui qui l'a fait vraiment connaître, permet alors de comprendre le fond de l'idéologie mandevillienne dans la Fable des abeilles: l'obtention de la plus grande richesse des nations possible repose sur le traitement séparé de deux sortes de «tricheurs» potentiels, d'un côté les entrepreneurs, puissants, juges... qu'il faut laisser abuser leur «clientèle », d'autre part les hommes de troupe et les ouvriers dans leur plus basse extraction qu'il faut empêcher de gruger leurs employeurs. 
Le raisonnement de Mandeville (qui, en psychiatre, regarde les hommes comme s'ils étaient dotés de symptômes) s'appuie sur l'idée selon laquelle les gens «honorables » (le sens de l'honneur étant transmis par l'éducation «choisie» des rejetons des classes aisées) tendent à relier un usage «élégant» de la richesse au fait d'afficher que l'on agit dans le sens du bien collectif, ce qui rend nécessaire d'avoir beaucoup de moyens :

Rien n'est plus universellement charmant que l'argent; il convient à tous les rangs [... . alors que l'honneur a peu d'influence sur les gens vils et qui se tuent de travail, et affecte rarement quelqu'un de vulgaire; mais alors, l'argent achète presque partout de l'honneur; la richesse même est en soi un honneur pour tous ceux qui savent en user élégamment. L'honneur au contraire a besoin de richesse pour se soutenir; sans elle, c'est un poids mort [...] Car plus on est une personne de qualité, plus la vie donne de besoins; mais plus on a d'argent, plus on est en état de fournir à leurs demandes les plus folles. (Mandeville, Cléomène $6^{e}$ dialogue, p. 289)

Les hommes «bien» ne peuvent pas de ce fait s'empêcher d'adopter des manières des plus finement luxueuses pour impressionner les autres par leur apparence «élégante ». C'est à celui qui aura le plus beau carrosse, le logement le plus étonnant, les vêtements les plus fameux, les œuvres d’art les plus recherchées, l'épouse de la meilleure mine, la fonction la plus utile, qui proposera les repas les plus fins, qui aura fait les voyages les plus somptueux, qui réunira le plus souvent ses connaissances ${ }^{15} \ldots$ Ces gens feront souvent beaucoup d'effort, en trompant parfois sans problème d'autres hommes, pour s'enrichir et avoir les moyens de ce désir de gloire :

Ce qui avance et encourage les arts et les sciences, c'est leur récompense, l'argent et les honneurs [...] L’ambition, la cupidité et souvent la nécessité sont souvent de puissantes incitations au travail et à l'application; et elles arrachent souvent à la paresse [...] des hommes sur qui les arguments ou les châtiments de leur père ou de leur percepteur n'avaient fait aucune impression dans leur jeunesse. (Mandeville, Cléomène 6e dialogue, p. 280)

I5 Rousseau ne cessera pas d'implorer les hommes pour qu'ils ne cèdent pas aux tentations de l'amour propre (rêver par exemple d'être un grand capitaine au mépris des hommes de troupe servant de chair à canons, de vivre dans le luxe sans se soucier de la main d'œuvre servile et misérable qui permet l'enrichissement...). 
Ces individus «comme il faut» sont pour Mandeville très productifs pour l'ensemble de la collectivité car ils ne cessent pas alors de créer de nouvelles activités (par exemple de renouveler les modes et les styles) et d'utiliser avec soin leurs ressources pour avoir le plaisir d'étaler leur «noble» réussite :

C'est ainsi que le vice entretenait l'esprit d'invention qui, joint au temps et à l'industrie, avait porté les commodités de l'existence, ses plaisirs [...] à un tel point que les pauvres eux-mêmes vivaient mieux que les riches auparavant (Mandeville, Le Poème, p. 34) - associé à la Remarque O (p. II8-II9): À sa table il demande de nombreux services, chacun fait d'une variété choisie de mets exquis, difficiles à se procurer, et qui soient le témoignage achevé d'une cuisine recherchée et du meilleur goût, tandis qu'une musique harmonieuse et des flatteries [...] charment tout à tour ses oreilles [...] Il ambitionne de vastes palais et des jardins délicieux ; son plus grand plaisir c'est de surpasser les autres avec ses nobles chevaux, ses carrosses magnifiques, sa suite nombreuse, et son mobilier coûteux [...] il lui faut des femmes de bon ton [...] Il n'emploie [...] que les ouvriers les plus capables et les plus habiles, afin de faire voir son jugement et son goût.

Leurs lois et leurs habits étaient sujets à variations car ce qui un temps était fort bien en six mois devenait un crime (Mandeville, Le Poème, p. 34) - associé à la Remarque M (p. I05) : car cette émulation, ces efforts continuels pour l'emporter les uns sur les autres qui font que, après tous ces changements [...] dans les modes, où on en invente de nouvelles et on en renouvelle d'anciennes, il reste encore un plus ultra pour les gens ingénieux. C'est elle, ou du moins ce sont ses conséquences, qui donnent du travail aux pauvres, stimulent l'industrie, et encouragent l'ouvrier habile à chercher encore des perfectionnements.

L’arrogante Chloé, afin de vivre sur un grand pied, avait obligé son mari à piller l’État. Mais voici qu'elle vend ses meubles, pour lesquels on avait dépouillé les Indes; elle réduit sa table qui lui coûtait cher, et porte son vêtement d'usage une année entière $[. .$.$] Les tisserands, qui à la riche soie joignaient les métaux$ précieux, et tous les métiers accessoires ont disparu [...] Ce ne sont plus les négociants, mais les compagnies qui suppriment des manufactures entières. Le contentement, ruine de l'industrie, les remplit d'admiration pour l'abondance de biens tout simples sans en chercher ou en désirer davantage. (Mandeville, Le Poème, p. 38-39, sur les suites de la renonciation au vice) 
Ainsi, alors que chez les moralistes comme La Rochefoucauld, la recherche des signes de la richesse par les hommes «comme il faut» nuisait à la richesse des nations ${ }^{16}$ (comme si l'argent détourné dans le luxe et l'arrivisme n’était pas placé là où il devait l'être), elle la favorise chez Mandeville. Il admet autrement dit qu'il ne faut pas s'inquiéter du caractère parfois «abject» des motivations des employeurs, médecins, avocats, puissants... puisqu'ils favorisent de toute façon la croissance économique ${ }^{17}$. Pourtant, ces gens «comme il faut» qui parviennent si bien à s'enrichir ne cessent souvent d'exploiter hypocritement d'autres hommes sans vraie pitié pour les plus pauvres ${ }^{18}$, ces derniers se faisant tout bonnement berner :

d'autres étaient condamnés à la faux et à la bèche, et à tous ces métiers pénibles et laborieux où jour après jour s'échinent volontairement des misérables épuisant leurs forces et leur santé pour avoir de quoi manger [...], ces saints hommes de peine mourraient de faim [...], les pauvres et les gens réduits à l'extrémité [...], poussés par la seule nécessité, se trouvaient attachés au bois sinistre pour des crimes qui ne méritaient pas ce sort. (Mandeville, Le Poème, p. 30-33)

16 «Le luxe et la trop grande politesse dans les États sont le présage assuré de leur décadence parce que tous les particuliers s'attachant à leurs intérêts propres, ils se détournent du bien public.» (La Rochefoucauld, Maxime 53 retranchée après la Ire édition, p. 18I)

17 Mandeville postule aussi que les dépenses de police, justice... participent à la richesse des nations parce qu'elles accroissent le niveau d'activité. Voir le dilemme des gredins dans l'Essai sur la charité et les écoles de charité p. 216: «Les gredins ont les mêmes passions à satisfaire que les autres hommes, et s'estiment eux-mêmes selon leur honneur et leur loyauté les uns envers les autres, leur courage.»

I8 Rousseau, bien qu'il s'oppose à Mandeville en expliquant que la morale véritable est en fait le produit de la pitié, affirmera ainsi que le sauvage et la populace ont meilleur fond que les «honnêtes gens»: «Mandeville a bien senti qu'avec toute leur morale les hommes n'eussent jamais été que des monstres, si la Nature ne leur eût donné la pitié à l'appui de la raison: mais il n'a pas vu que de cette seule qualité découlent toutes les vertus sociales qu'il veut disputer aux hommes [...] Dans les Émeutes, dans les querelles des Rues, la populace s'assemble, l'homme prudent s'éloigne: c'est la canaille, ce sont les femmes des Halles, qui séparent les combattants, et qui empêchent les honnêtes gens de s'entr'égorger. II est donc bien certain que la pitié est un sentiment naturel, qui modérant dans chaque individu l'activité de l'amour de soi même, concourt à la conservation mutuelle de toute l'espèce.» (Rousseau, 1755) 
Par contre, Mandeville explique qu'il faut tout faire pour que les hommes de troupe et les ouvriers dans leur plus basse extraction ne grugent pas leurs employeurs et qu'ils restent ainsi une main d'œuvre servile prête à effectuer bravement les travaux les plus ingrats et d’ardents combattants quand la société a besoin de chair à canon ${ }^{19}$. Donnant une importance considérable aux modalités du système éducatif qui doit selon lui être dual ${ }^{20}$, il recommande même de ne pas offrir aux enfants des pauvres la possibilité d’accéder aux écoles de charité, c'està-dire aux institutions susceptibles d'éveiller leur esprit critique par la formation. Mandeville s'est rendu célèbre à ce propos en présentant dans son Essai sur la charité et les écoles de charité la pitié (dont il reconnaît expressément l'existence) que ressentent les hommes envers les enfants des misérables comme contrariant le processus de création de la plus grande richesse nationale possible:

Il est clair que dans une nation libre où l'esclavage n'est pas autorisé, la richesse la plus sûre consiste à avoir une multitude de pauvres travailleurs; car outre que c'est là une pépinière intarissable pour les flottes et les armées, sans eux on ne pourrait jouir de rien, et aucun produit d'aucun pays n'aurait de valeur

Où trouverons-nous meilleure pépinière de ces êtres nécessaires que parmi les enfants de pauvres? [...] ce qui les rend odieuses [ces vérités], c'est une disposition à un misérable aspect religieux pour les pauvres [...], et qui nait d'un sentiment de pitié, de sottise (Mandeville, Essai sur la charité et les écoles de charité, p. 243 et p. 225)

Ainsi, s'il existe bien chez Mandeville un mécanisme ressemblant à la «main invisible» puisque l'action individuelle intéressée a effectivement des conséquences non intentionnelles bénéfiques (nombre de pauvres vivant mieux en général quand les riches «comme il faut» se laissent aller à leur désir de gloire et de richesses), il précise qu'une fraction de la population doit et restera toujours misérable et servile pour se charger des tâches les plus pénibles: les «lawgivers and other wise men » se chargent d'assurer d'une manière ou d'une autre la permanence d'un tel volant de main d'œuvre. Pour Mandeville, la civilisation ceux des autres types d'acteurs sont la véritable cause du chômage prolifèrent aujourd'hui (voir les théories du salaire d'efficience type modèle du tire-au-flanc, la théorie insiders-outsiders...). Voir Michéa (1999) p. 89-95 qui s'inspire de Christopher Lasch. 
doit ainsi sa naissance et son expansion à l'existence d'un péché (le sort funeste réservé aux plus pauvres), sachant qu'il n'existe aucun moyen d’y échapper parce que, si l'on assure la promotion intellectuelle des enfants de misérables, il faudra toujours que les «lawgivers and other wise men » en trouvent d'autres pour les remplacer.

Adam Smith, après avoir signalé dans ses premières publications la proximité paradoxale des systèmes de Mandeville et de Rousseau si l'on met de côté le rôle conféré à la pitié et s'être fait le grand dénonciateur du système présenté dans la Fable des abeilles, se montrera d'une ingéniosité extrême en affirmant (Smith, 1759) que les propriétaires fonciers sont certes bien souvent des gens faussement «comme il faut» emplis d'un médiocre désir de luxe et de gloire, mais qu’ils sont pourtant conduits par une main invisible à accomplir presque la même distribution des nécessités de la vie que celle qui aurait eu lieu si la terre avait été divisée en portions égales entre tous ses habitants si bien qu'ainsi, sans le vouloir, ils servent les intérêts de la société et donnent des moyens à la multiplication de l'espèce. En reprenant le même type de logique quant à la relation entre employeurs et ouvriers dans la Richesse des nations (I776), il parviendra à gommer dans nombre d'esprits que, chez Mandeville, la main invisible réservait quand même un sort inexorablement funeste à une partie de la population, celle des sacrifiés pour l’avantage soi-disant commun, en fait pour celui des gens faussement «comme il faut» au premier plan. Smith a réussi autrement dit à transformer la main invisible de Mandeville en lui retirant l'idée selon laquelle la civilisation s'est construire et développée, telle une réaffirmation permanente du péché originel, sur le «sacrifice» permanent d’une partie de la population.

\section{CONCLUSION}

Pour Mandeville, les hommes se seraient régulés d'eux-mêmes à première vue en créant et intégrant au début de la civilisation la fameuse distinction entre «vil» et «noble» qui vient donner du plaisir à ceux qui paraissent se comporter «comme il faut» compte tenu des applaudissements reçus pour leur bonne attitude. Cette distinction est ce qui a permis le développement au dedans des individus d'un orgueil régulé les poussant à agir apparemment dans le sens du bien collectif. Mais Mandeville ajoute un second niveau de 
raisonnement infiniment plus caustique : nombre d'hommes dans leurs relations mandatés / mandataires ont en fait tendance à vouloir se gruger les uns les autres, cet aspect de la nature humaine ayant favorisé la mise en place de la superstructure morale parce qu'elle permet de faire croire à sa «clientèle» que l'on se comporte «comme il faut» et de mieux s'organiser pour spolier son prochain. La clé des progrès de la civilisation tient sur cette base à ce que l'ordre moral et juridique permet les comportements «méchants» quand ils semblent se faire dans l'avantage du plus grand nombre et les interdit quand tel n'est pas le cas. Le point central de ce mécanisme fort efficace est alors qu'une fraction de la population se trouvera ainsi toujours cantonnée aux tâches les plus ingrates (les «lawgivers and other wise men » l'empêchant autant que possible de se comporter «méchamment»). La conséquence d'un tel processus spontané est que les pires des hommes, emplis d'un gigantesque désir d’enrichissement et d’applaudissement et prêts à tout pour atteindre hypocritement un tel objectif, ont tendance à s'en sortir toujours mieux que les autres et à occuper les meilleures places (sachant que les gens qui «réussissent» ne sont pas tous des «plus méchants»).

\section{BIBLIOGRAPHIE}

AXELROD Robert, 1984, The Evolution of Cooperation, N.Y.C., Basic books.

BAYLE Pierre, 1682, Pensées diverses écrites à un docteur de Sorbonne à l'occasion de la Comète qui parut au mois de décembre 1680 , tome second, Rotterdam, Reinier Léers.

BENTHAM Jeremy, BROWING John, 1834, Deontology and the science of morality, 2 volumes, domaine public.

BINMORE Ken, 2005, Natural justice, Oxford, Oxford University Press.

-, 2007, Playing for real: a text on game theory, Oxford, Oxford University Press.

-, 2009, Game theory, dans Peil J. et Van Staveren I. (dir.), Handbook of economics and ethics, Northampton, MA., Edward Elgar Publishing. 
BRIDEL Pascal, 2009, «Passions et intérêts revisités: la suppression des "sentiments" est-elle à l'origine de l'économie politique?», Revue européenne des sciences sociales, XLVII, I44, p. I35-I50.

CAILLÉ Alain, SENELLART Michel, LAZZERI Christian (dir.), 2007, Histoire raisonnée de la philosophie morale et politique, 2 tomes, Paris, Flammarion.

CARRIVE Paulette,1980, Bernard Mandeville: passions, vices, vertus, Paris, Vrin.

CASTEL DE SAINT-PIERRE Charles-Irénée, I74I, Ouvrages de morale contre l'opinion de Mandeville, dans Ouvrages de morale et de politique, Rotterdam, Jean Daniel Beman.

DELLEMOTTE Jean, 2009, «La main invisible d'Adam Smith: pour en finir avec les idées reçues», L'économie politique, n44, p. 28-41.

DOCKÈS Pierre, 2008, Hobbes: économie, politique et terreur, Paris, Economica.

DOMENECH Jacques, 1989, L'Éthique des Lumières: les fondements de la morale dans la philosophie française du XVIII siècle, Paris, Vrin.

ESPRIT Jacques, 1677-1678, La Fausseté des vertus humaines, 2 tomes, Paris.

GROTIUS Hugo, 2005 [1625], Le Droit de la guerre et de la paix, Paris, PUF, «Quadrige».

HARDIN Russel, 2007, David Hume: moral and political theorist, Oxford, Oxford University Press.

HAYEK Friedrich, 1966, "Lecture on a Master Mind: Dr. Bernard Mandeville", Proceedings of the British Academy, vol. 52, p. 125-141.

HELVÉTIUS, 1758, De l'esprit, domaine public.

HIRSCHMAN Albert, 1980 [1977], Les Passions et les Intérêts, Paris, PUF, «Sociologies》.

HUME David, 1993 [1740], La Morale, livre 3 du Traité de la nature humaine, Paris, GF Flammarion.

-, 1991 [1751], Enquête sur les principes de la morale, Paris, GF Flammarion.

-, 200I (divers XVIII siècle), Essais moraux, politiques et littéraires et autres essais, Paris, PUF, «Perspectives anglo-saxonnes». 
HUTCHESON Francis, 2003 [1728], Essai sur la nature et la conduite des passions et affections avec illustrations sur le sens moral, Paris, L'Harmattan, «Ouvertures philosophiques ».

LAFOND Jean, 1990, Mandeville et La Rochefoucauld, ou des avatars de l'augustinisme, dans Gestaltung-Umgestaltung: Beiträge zur Geschichte der romanischen Literaturen, Tübingen, Gunter Narr, p. I37-I50.

LA ROUCHEFOUCAULD François (de la), 2007 [1664], Maximes, Paris, Le livre de poche.

LE JALLÉ Éléonore, 2003, «Hayek lecteur des philosophes de l'ordre spontané: Mandeville», Hume, Ferguson, Astérion, nI, p. 88-III.

-, 2005, L'autorégulation chez Hume, Paris, PUF, «Pratiques théoriques».

-, 2009, «La convention de David Hume à David Lewis», dans Gautier C., Laugier S. (dir), Normativités et sens commun, Paris, PUF.

LEWIS David, 2002 [1969], Convention, Cambridge, Mass., Blackwell.

MANDEVILLE Bernard, 2007 [1705-1724], La Fable des abeilles, première partie, Paris Vrin, textes philosophiques, trad. Carrive L. et P.

-, 1998 [1723], Recherche sur la nature de la société, addition à la seconde édition (1723) de la Fable des abeilles, Arles, Actes Sud, «Babel», trad. Carrive L. et P. -, 1998 [1729], La fable des abeilles, deuxième partie, Paris, Vrin, «Textes philosophiques», 1998, trad. Carrive L. et P.

MICHÉA Jean-Claude, 1999, L'Enseignement de l'ignorance, Paris, Climats.

NÉMO Philippe, 1988, La Société du droit selon F-A Hayek, Paris PUF.

NICOLE Pierre, 1999 [167|-1675], Essais de morale, Paris, PUF.

POSNER Eric, 2007, Social norms, nonlegal sanctions, and the law, Northampton, MA., Edward Elgar Publishing.

POSTEL Nicolas, SOBEL Richard, 2006, «Quelle théorie hétérodoxe de l'acteur économique?», dans Eymard-Duvernay F. (dir.), L'Économie des conventions, tome I, La Découverte, p. 31-150.

RAWLS John, 2002, Leçons sur les origines de la philosophie morale, Paris, La Découverte. 
ROUSSEAU Jean-Jacques, 1755, Discours sur l'origine et les fondements de l'inégalité parmi les hommes, domaine public.

SALAIS Robert, 2009, «Conventions de travail, mondes de production et institutions: un parcours de recherche», L'Homme et la Société, 170-17I, p. 151-174.

SMITH Adam, 2007 [1759], Théorie des sentiments moraux, PUF, « Quadrige».

STEWART Dugald, 1828-1834, Philosophie des facultés actives et morales de l'homme, tome 2, Éditions Johanneau. 